\title{
Enhanced emission from Si-based light-emitting diodes using surface plasmons
}

\author{
S. Pillai, ${ }^{a)}$ K. R. Catchpole, T. Trupke, G. Zhang, J. Zhao, and M. A. Green \\ Centre of Excellence for Advanced Silicon Photovoltaics and Photonics, University of New South Wales, \\ Sydney, 2052, Australia
}

(Received 14 December 2005; accepted 18 March 2006; published online 17 April 2006)

\begin{abstract}
Excitation of surface plasmons on metallic nanoparticles has potential for increasing the absorption and emission from thin Si devices. We report an eight-fold enhancement in electroluminescence from silicon-on-insulator light-emitting diodes at $900 \mathrm{~nm}$ via excitation of surface plasmon resonance in silver nanoparticles, along with a redshift in the electroluminescence by $70 \mathrm{~nm}$ by overcoating the nanoparticles with $\mathrm{ZnS}$. The enhancement is due to coupling between the electromagnetic excitations of the silver nanoparticles and the waveguide modes. (C) 2006 American Institute of Physics. [DOI: 10.1063/1.2195695]
\end{abstract}

The need to integrate optical functionality into computer chips, e.g., for faster data communication is attracting a lot of research related to the development of integrated lightemitting diodes (LEDs) or lasers. Si is a strong contender in spite of it being an indirect band-gap material as it is cheaper and easier to integrate into Si microchips. ${ }^{1}$ The luminescence quantum efficiency for band edge light emission from Si can be improved by surface passivation and light trapping, as was demonstrated in record efficiency wafer-based bulk $\mathrm{Si}$ LEDs. ${ }^{2}$ However, for thin films, light trapping using the conventional technique of surface texture with features which are typically around 10 microns in size, would be at the cost of the already thin semiconductor layer and would cause increased recombination losses. In this work, we propose the use of nanoscale metal particles as an effective method for increasing light emission as well as light absorption in thinfilm Si devices.

Surface plasmons are the collective oscillation of free electrons in a metal. They occur at the interfaces between metals and dielectrics and decay exponentially with distance from the interface. ${ }^{3}$ Because of the large free electron density of metals, they show strong resonances at optical frequencies. Deposition of gold nanoparticles showed increased absorption from a $p n$ junction fabricated on a Si wafer ${ }^{4}$ and an eighteen-fold increase in the photocurrent at $800 \mathrm{~nm}$ from silicon-on-insulator (SOI) photodetectors coated with silver nanoparticles was reported, ${ }^{5}$ both effects being assigned to the effect of surface plasmons. The plasmon effect can be used not only to enhance photocurrent from devices, but also to increase the efficiency with which internally generated photons are extracted from the waveguide in light-emitting diodes. ${ }^{6}$ The surface plasmon resonance frequency for nanospheres is given by

$$
\omega_{\mathrm{sp}}=\frac{\omega_{p}}{\sqrt{1+2 \varepsilon_{m}}},
$$

where $\omega_{p}$ is the plasma frequency and $\epsilon_{m}$ is the permittivity of the medium which can be taken as the average ${ }^{7}$ of the substrate and the surrounding medium and written as $\epsilon_{\mathrm{av}}$ $=\left(\epsilon_{\text {sub }}+\epsilon_{\text {ext }}\right) / 2$. From Eq. (1), it can be clearly seen that by varying $\epsilon_{m}$ the surface plasmon frequency can be tuned, and that increasing the permittivity of the medium leads to a

${ }^{a)}$ Electronic mail: supriyap@student.unsw.edu.au redshift of the plasmon resonance. Shifting the plasmon resonance has been demonstrated by overcoating or sandwiching the metal particles in a high refractive index medium. ${ }^{7,8}$ Equation (1) is valid for thick substrates but has been used here in a general context to help understand the effect of dielectric overcoating.

The basic device structure used here is shown schematically in Fig. 1. It consists of a lateral $p n$ junction fabricated on SOI substrates. We deposit metal nanoparticles on the SOI structure and use the coupling of the broad surface plasmon resonance and the waveguide modes to scatter internally emitted light out of the silicon. Overcoating the islands with $\mathrm{ZnS}$ is then used to redshift the surface plasmon resonance. The enhancement in the emission and absorption properties are studied using electroluminescence (EL) and spectral response (SR) measurements, emphasizing the potential of surface plasmons for both enhanced emission and absorption, respectively.

The layers of the device structure are: A $35 \mathrm{~nm}$ thick passivating top oxide layer which acts as a spacer layer as well, $95 \mathrm{~nm} \mathrm{Si}$ active layer and $400 \mathrm{~nm}$ thick buried oxide layer. Because the total recombination in SOI devices is dominated by the surface recombination the passivation of the top silicon layer is imperative but for maximum coupling between the silver particles and the $\mathrm{Si}$ waveguide the thickness of the top oxide layer is kept at the minimum thickness required for good passivation. The $p$ and $n$ metal fingers are interdigitated on the front surface for maximum carrier injection. A thin layer of $14 \mathrm{~nm}$ silver is deposited by vacuum evaporation at $3 \times 10^{-5}$ torr at the rate of approximately

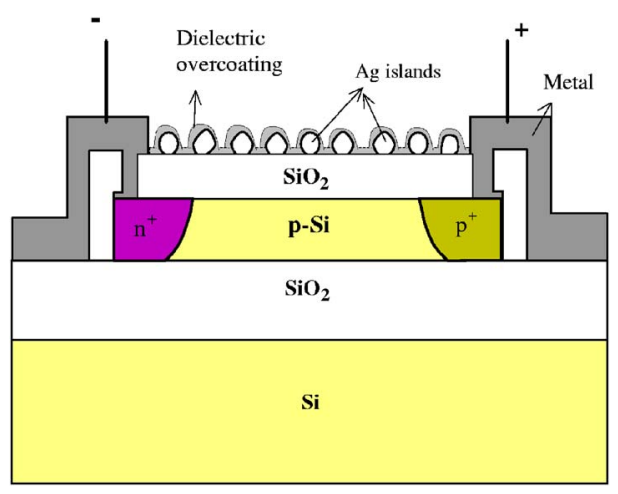

FIG. 1. (Color online) Schematic of SOI LED used in the experiments. 


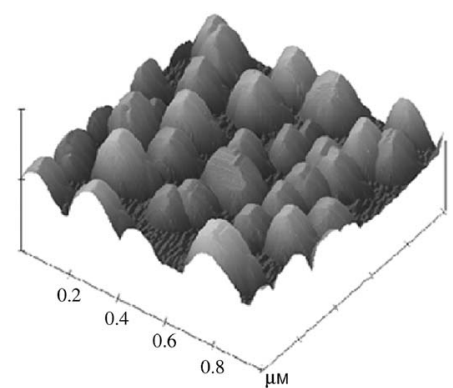

(a)

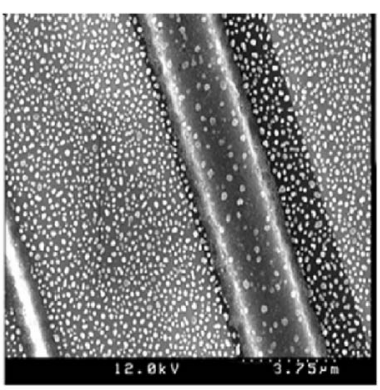

(b)
FIG. 2. (a) AFM image showing elevation of the metal particles. (b) SEM picture of the silver islands on the SOI LED.

$2 \AA /$ sec. The samples are then annealed in nitrogen at $200^{\circ} \mathrm{C}$ for $55 \mathrm{~min}$. The metal particles coalesce together to form metal islands. The shape and the size of the islands depend on the substrate, thickness of the evaporated layer and also on the annealing temperature and time. Analysis of SEM and AFM images (Fig. 2) using image processing software show the average particle size to be around $120-140 \mathrm{~nm}$ in diameter and around $45-60 \mathrm{~nm}$ in height. A thin layer of $30 \mathrm{~nm}$ $\mathrm{ZnS}$ was then evaporated on the metal islands at1 $\times 10^{-6}$ torr. The thickness was so chosen that the $\mathrm{ZnS}$ layer acts as a dielectric layer but does not introduce additional waveguide modes.

The "bare" island resonance for islands deposited on a microscope glass slide was measured separately using transmittance measurements for the cases with islands only and for the $\mathrm{ZnS}$ overcoated islands. Figure 3 shows a very evident redshift in the resonance at the dip in the transmittance measurement.

EL and spectral response of each device were measured before and after deposition of the metal islands and after deposition of the additional $\mathrm{ZnS}$ layer, respectively. A reference sample was used in each case to check for the repeatability of the measurements which were typically carried out several days apart.

Typical results of the EL measurements are shown in Fig. 4(a). The corresponding enhancement for the case with islands only and for the case with overcoated islands is shown in Fig. 4(b), where the enhancement is the ratio of the EL or photocurrent signal (Fig. 6) over the signal before island deposition. Our results show a factor of approximately 8 times enhancement in emission from the SOI LEDs at a wavelength of $900 \mathrm{~nm}$. We estimate the variations in the ex-

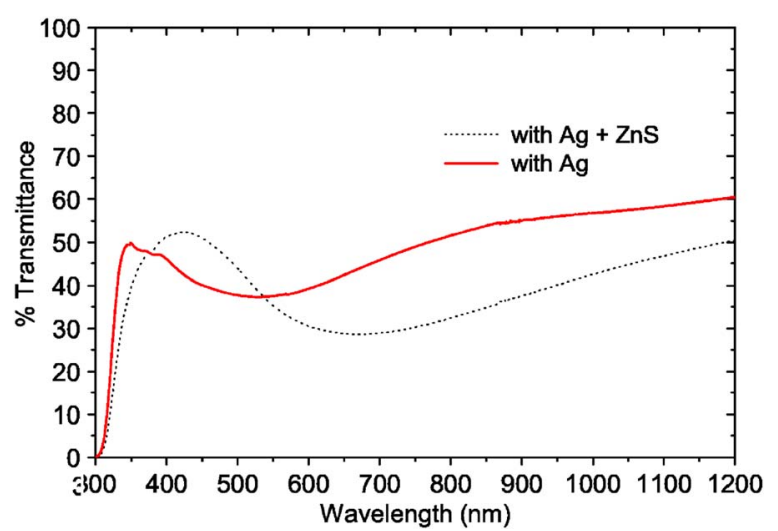

FIG. 3. (Color online) Shift in the bare island resonance using dielectric overcoating. hercoating.
Downloaded 08 Mar 2010 to 150.203 .243 .38 . Redistribution subject to AlP license or co
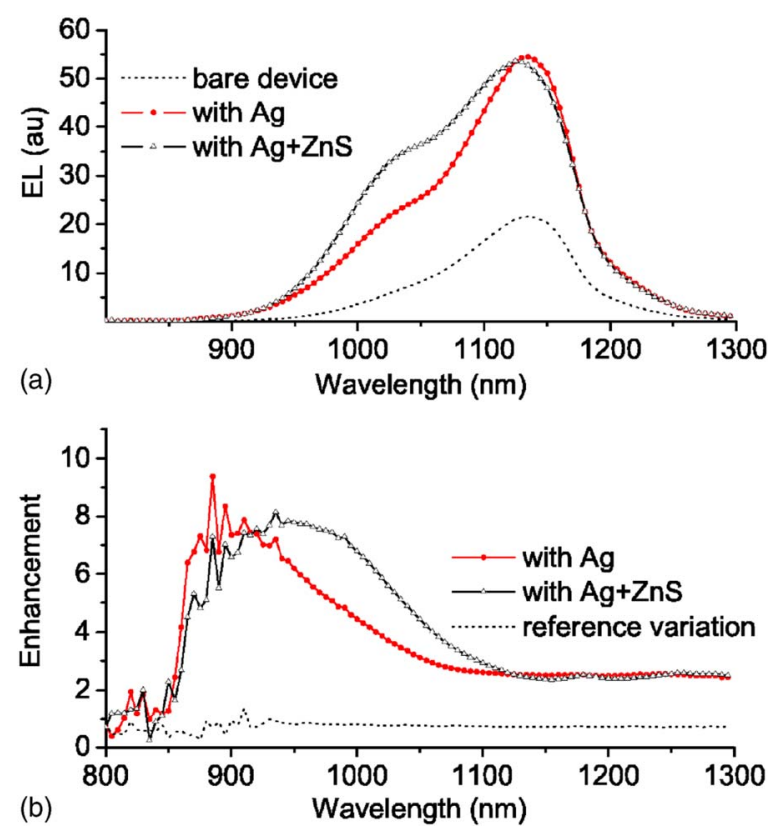

FIG. 4. (Color online) (a) EL plots before and after the deposition of silver islands and $\mathrm{ZnS}$ overcoating. (b) Enhancement plots for the data from (a) also showing the variation of the reference sample without the silver islands (dotted line).

perimental setup to be $<20 \%$ and hence the observed enhancement can clearly be assigned to the presence of the metal islands rather than to variations in the experimental conditions.

A further redshift and an overall enhancement in EL is observed after overcoating the metal nanoparticles with $\mathrm{ZnS}$, which is a dielectric layer of refractive index 2.2. The total enhancement integrated across the emission spectrum is a factor of 2.8 for uncoated islands, and 3.2 for overcoated islands.

We deposited two-thirds of a LED with silver islands and measured the EL from the LED. The emission was captured by a charge coupled device (CCD) camera and is shown in Fig. 5. There is clear evidence for the increase in intensity from the area with the nanoparticles. The average emission intensity is about five times higher from the area with metal islands. Taking into account the sensitivity of the CCD camera, which is more sensitive at a short wavelength where the enhancement is most pronounced, this figure is consistent with the data from Fig. 4. The enhancement for the photocurrent as calculated from the spectral response of a device is shown in Fig. 6.

According to a generalized form of Kirchoff's law, which is valid for luminescent emission, enhanced emittance

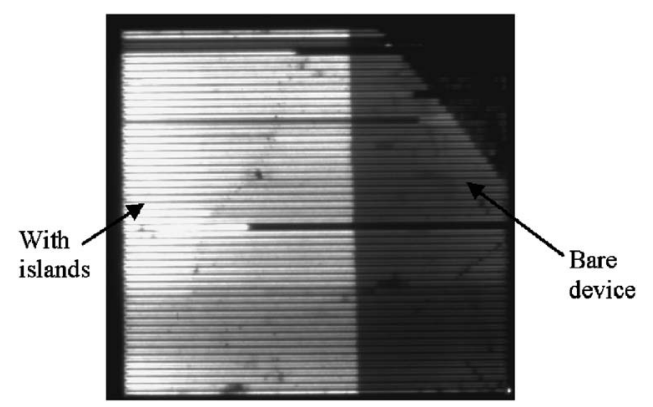

FIG. 5. EL image showing the difference in EL from one LED that is partially covered with silver islands (left). Brighter areas correspond to 


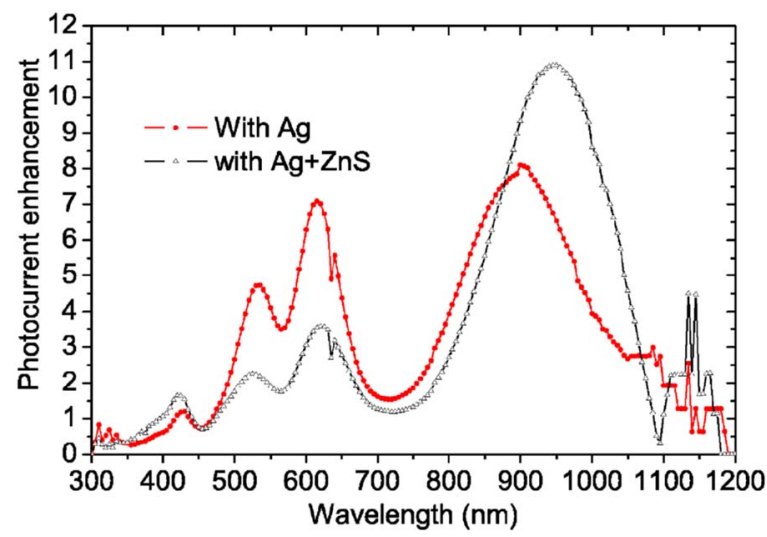

FIG. 6. (Color online) Photocurrent enhancement plots for the case with metal islands and for the case of metal islands overcoated with $\mathrm{ZnS}$.

corresponds to an enhanced absorption. ${ }^{9}$ Our SOI-LEDs can be used as either solar cells or LEDs. As we show here, measurements of the SR and of the luminescence on these devices complement each other as they provide information about the absorptance/emittance enhancement in different spectral ranges above and below the band gap, respectively, with a small spectral overlap region around the band-gap energy. The position of the peaks for the photocurrent and EL agree well along with the $70 \mathrm{~nm}$ redshift that is observed with the dielectric overcoating. An interesting observation from Fig. 6 is that the enhancement factor is larger than unity throughout the optical regime, which is a promising result for photovoltaic applications. Because of the thin layer of $\mathrm{Si}$ used, photon reabsorption is minimal and therefore the relative intensity in the emission at short wavelengths is higher when compared to bulk $\mathrm{Si}^{10}$ With the use of the dielectric overcoating layer, this short-wavelength effect is further enhanced by the shoulder as can be seen in Fig. 4(a).

The enhancement in emission and absorption occurs because the metal particles can excite surface plasmons and when they are in the near field of the waveguide they couple strongly with the waveguide modes. ${ }^{5}$ For emission, only a small fraction of the waveguided light is coupled out of the waveguide in each interaction with the metal nanoparticles, but because the band edge light is very weakly absorbed, the emission is significantly enhanced after multiple scattering events. The position of the peaks occur at the waveguide cut-off frequencies, ${ }^{11}$ but can be shifted somewhat by altering the bare island resonance of the nanoparticles. The scattering by the metal nanoparticles, which is proportional to the extinction, tends to decrease with wavelength in the ab- sence of the waveguide structure as shown in Fig. 3. If we consider the scattering when the waveguide is present to be a product of the bare island resonance and a factor describing the interaction of the nanoparticles with the waveguide modes, then we can see that redshifting the bare island resonance peak (by overcoating) will also redshift the scattering peaks in the combined system. The redshift is larger at long wavelengths, as is evident from the photocurrent enhancement peaks in Fig. 6. This is because the change in the bare island resonance with an overcoating is larger at long wavelengths.

A major advantage of the surface plasmon approach is that, unlike texturing of the silicon, the application of the metal nanoparticles-which are separated from the silicon by a thin spacer layer-will not degrade the electrical characteristics of the device. The metal islands are deposited by a simple process of evaporation and low-temperature annealing, and can be formed as the last stage of the device processing so there are no processing incompatibilities.

We demonstrate an eight-fold increase in the EL signal at $900 \mathrm{~nm}$, and an overall factor of 3 enhancement from silicon-based devices using a very simple deposition process. We also report the use of overcoating the surface plasmon resonance to redshift and increase the enhancement. This is a very promising result with the potential to not only increase light emission from thin films of varying substrates but to increase the photocurrent from solar cells.

The Centre of Excellence for Advanced Silicon Photovoltaics and Photonics is supported under the Australian Research Council's Centres of Excellence Scheme.

${ }^{1}$ P. Ball, Nature (London) 409, 974 (2001).

${ }^{2}$ M. A. Green, J. Zhao, A. Wang, P. J. Reece, and M. Gal, Nature (London) 412, 805 (2001).

${ }^{3}$ H. Raether, Surface Plasmons on Smooth and Rough Surface and on Gratings (Springer, Berlin, 1988).

${ }^{4}$ D. M. Schaadt, B. Feng, and E. T. Yu, Appl. Phys. Lett. 86, 063106 (2005).

${ }^{5}$ H. R. Stuart and D. G. Hall, Appl. Phys. Lett. 73, 3815 (1998).

${ }^{6}$ W. L. Barnes, J. Lightwave Technol. 17, 2170 (1999).

${ }^{7}$ G. Xu, M. Tazawa, P. Jin, S. Nakao, and K. Yoshimura, Appl. Phys. Lett. 82, 3811 (2003).

${ }^{8}$ H. Mertens, J. Verhoeven, and A. Polman, Appl. Phys. Lett. 85, 1317 (2004).

${ }^{9}$ P. Wurfel, J. Phys. C 15, 3967 (1982).

${ }^{10}$ J. Zhao, G. Zhang, T. Trupke, A. Wang, F. Hudert, and M. A. Green, Appl. Phys. Lett. 85, 2830 (2004).

${ }^{11}$ B. J. Soller and D. G. Hall, J. Opt. Soc. Am. A 18, 2577 (2001). 